\title{
Major vascular injury during minimally invasive gynecologic surgery: simulation-based approach to teaching surgical crisis management skills
}

\section{Cara R. King, ${ }^{\text {a }}$ Annie Kim, ${ }^{\mathrm{b}}$ Nicole Donnellan ${ }^{\mathrm{c}}$ and Veronica Lerner ${ }^{\mathrm{b}, *}$}

${ }^{a}$ Obstetrics, Gynecology, and Women's Health Institute, Section of Minimally Invasive Gynecologic Surgery, Cleveland, OH, USA; ${ }^{b}$ Department of Obstetrics \& Gynecology and Women's Health, Albert Einstein College of Medicine/Montefiore Medical Center, Bronx, NY, USA; 'Obstetrics, Gynecology and Reproductive Sciences, Magee-Womens Research Institute, UPMC Magee-Womens Hospital, Pittsburgh, PA, USA

${ }^{*}$ Corresponding author at: Montefiore Medical Center/Albert Einstein College of Medicine, 1300 Morris Park Ave, Block 632, Bronx, NY 10461, USA. Email: lernervt02@gmail.com

Date accepted for publication: 27 October 2021

\section{Abstract}

Background: Major vascular injury (MVI) in gynecologic surgery is a rare catastrophic complication. We developed and implemented MVI simulation and conducted it at the 2019 AAGL Fellowship in Minimally Invasive Gynecologic Surgery (FMIGS) Annual Bootcamp. Postgraduate year (PGY) 6 fellows participated as learners. FMIGS faculty conducted the simulation. Methods: We developed a scenario, built a simulated abdomen including pelvic structures, designed the simulation setup given available space and resources, and created schedules for participant flow. Participants were expected to recognize an MVI that occurred during laparoscopic entry, resuscitate the patient, perform compression of the bleeding vessel, and call for a vascular surgery consult for vascular repair. A workshop was conducted for course faculty 1 week before the event and a rehearsal session was performed 1 day before the live simulation for all faculty. Participating fellows were divided into groups of 10, each session lasted 90 min and consisted of pre-briefing, simulation, and a debrief. Pre- and post-simulation survey responses were compared using McNemar's test. Median and interquartile ranges were calculated on responses collected via a Likert scale (1-5). Results: A total of 34 fellows (median age, 31.5 years) and 8 faculty (median age, 37.5 years) responded. Fellows reported a median of $8 \mathrm{~h}$ per year spent participating in simulation team training and a median of $5 \mathrm{~h}$ per year spent teaching in this context. All fellows believed that simulation training is an essential part of clinical practice and patient safety; $94.1 \%(n=32)$ thought that simulation team training should be a part of the boot camp; $94.1 \%(n=32)$ also thought that the training should be part of the fellowship curriculum at their respective institutions. Before the simulation, $97.1 \%(n=33)$ of the fellows desired to learn more about simulation training and curriculum development. After the simulation, all fellows felt that knowledge gained could be transferred to the clinical setting, felt more confident in responding to a critical intraoperative event, learned techniques to communicate more effectively during a critical event, and felt that the simulation content was relevant to their training and clinical practice. Most of the fellows felt more confident in managing intraoperative hemorrhage (median, 5; interquartile range, 4-5) after the simulation. Conclusion: MVI training in gynecologic surgery is feasible and viewed favorably by participants. Further work should focus on performance assessment and clinical outcomes to allow for the growth of simulation training within the field of gynecology.

Keywords: major vascular injury; simulation; minimally invasive gynecology fellows; surgical bootcamp

\section{Introduction}

Major vascular injury (MVI) during laparoscopic surgery is a rare complication and is associated with high patient

This abstract was selected for presentation at the SGS 46th Annual Scientific Meeting in Jacksonville, Florida, March 29-April 1, 2020. ID\# 3272611. Meeting was canceled due to Coronavirus pandemic. mortality. This complication requires quick and efficient coordination of care for patient optimization, which may be difficult to execute secondary to the rarity of this event and significant stress on the surgeon. ${ }^{1,2}$ Research has shown that teams are often unprepared to handle crises. The inability to manage surgical complications quickly and efficiently 
can lead to significant mortality. ${ }^{3,4}$ Crisis resource management (CRM) and teamwork principles popularized by TEAM STEPPS provide the basis for efficient functioning during an emergency. Furthermore, the medical knowledge required to manage MVI and hemorrhage has been described recently. ${ }^{1,5,6}$

Simulation has been increasingly included in training and in the professional maintenance of skills for obstetricians and gynecologists over the last two decades. The science of simulation is advanced in some specialties and disciplines, including obstetric training. However, there has been scant application of these educational advances within the realm of gynecologic surgery. Specifically, simulation training on how to manage emergencies in the operating room has yet to gain visibility. ${ }^{7}$ To date, no work has been done on this topic in gynecologic surgery, and, in particular, in minimally invasive gynecologic surgery (MIGS). ${ }^{2,8}$

The aims of this study were to develop and describe an MVI simulation scenario in MIGS and to assess the experience of fellows and faculty with this simulation.

\section{Methods}

\section{Subjects and study design}

AAGL (Elevating Gynecologic Surgery, founded as American Association of Gynecologic Laparoscopists) is the main professional society of MIGS surgeons and the governing body of a 2-year fellowship in the USA. FMIGS (Fellowship in Minimally Invasive Gynecologic Surgery) leadership produces an annual surgical course for MIGS fellows ("boot camp"). Core faculty developed an MVI simulation to teach intraoperative crisis management skills and conducted this simulation at the August 2019 boot camp. Postgraduate year (PGY) 6 MIGS fellows attending the 2019 course participated in this simulation.

\section{Planning phase}

Three core faculty (VL, CK, ND) developed the scenario, built a simulated abdomen including pelvic structures, designed the simulation setup given available space and resources, and created schedules for participant flow. Core faculty recruited and trained assistant faculty, who served as embedded persons during the scenario and participated in the post-simulation briefing. Lead faculty $(\mathrm{VL})$ is a graduate of the Center for Medical Simulation 1-week instructor course, followed by 10 years of experience as a simulation training instructor and functioned as primary debriefer and facilitator. She also trained second lead faculty (CK) to conduct a simulation session in a standardized way according to Promoting Excellence and Reflective Learning in Simulation (PEARLS) and the advocacy/inquiry model in a series of one-on-one sessions and rehearsals in order to be able to run two sessions in parallel to accommodate the scheduling requirements of the course. ${ }^{9,10}$ A workshop was conducted for course faculty 1 week before the event (via telelearning) on simulation, PEARLS, and advocacy/inquiry debriefing. Telelearning was performed due to the variable amount of simulation experience of additional faculty as well as various geographic locations. In addition to the workshop, a rehearsal session was performed 1 day before the live simulation for all faculty. The rehearsal included a review of debriefing techniques as well as the assignment of primary and secondary debriefing roles. MVI educational materials were prepared by the core faculty (VL, CK, ND) and a vascular surgeon who served as the content expert. Faculty pre-reading and participant post-session reference materials (emailed after the session) are listed in Appendix 1. Learning objectives are listed in Box 1.

Due to the small-group hands-on format, all participating fellows were divided into groups of 10. Each group was led by two trained faculty instructors who managed the scenario and moderated the debrief and three additional faculty who participated as embedded team members. Each session lasted $90 \mathrm{~min}$ and consisted of pre-briefing, simulation, and a debrief. The timeline for the session is listed in Table 1. Pre- and post-simulation feedback surveys were collected from participants and faculty to assist with course assessment and improvement (Supplementary Material).

\section{Simulated operating room}

\section{Simulation environment}

The location of the annual boot camp course varies from year to year. During the August 2019 course, at the time of this study, the allotted space for the simulation was an empty room with no available simulation equipment. We had to organize the setup and flow of the course and make modifications to the simulation. All details outlined in this article reflect the simulation as conducted in August 2019. However, this simulation can be easily adapted to a simulation laboratory setting where a fully or partially equipped simulated operating room (OR) is available, inclusive of OR table, tower and monitors, simulated abdomen, vital signs monitor, and control room).

A room of approximately $9 \times 4.5 \mathrm{~m}$ was used and set up as pictured in Figs. 1 and 2. Due to the lack of control and observation rooms with one-way mirrors and microphones, we opted to use the simulation concept of "invisible wall." Tape was placed on the floor to help with a visual 
Box 1. Learning objectives

General:

- Demonstrate crisis resource management skills

- Communication

- Leadership

- Situational awareness

- Mutual support

Case-specific:

- Recognize and understand the management of intraoperative major vascular injury (MVI)

- Demonstrate appropriate initial management to control MVI

- Recognize deterioration in hemodynamic status

- Understand role as an intraoperative consultant

- Demonstrate fluid resuscitation

- Initiate massive transfusion protocol

- Call for vascular consult and equipment

- Prepare for laparotomy

- Hemorrhage control: apply pressure to control bleeding vessel and avoid improper surgical techniques

Table 1. Timeline for the 90 -min simulation session

\begin{tabular}{ll}
\hline Time & Content \\
\hline $10 \mathrm{~min}$ & Introduction \\
$15 \mathrm{~min}$ & Simulation \\
$55 \mathrm{~min}$ & Debriefing \\
$10 \mathrm{~min}$ & Wrap-up/course evaluation \\
\hline
\end{tabular}

representation of the "wall". This concept is utilized in lowtechnology settings; those behind the wall (faculty and observers) are not allowed to interact with active participants in the scenario. One end of the room was set up as pre-briefing and debriefing space, including chairs, arranged in a circle to optimize participant interaction.

\section{Equipment}

Equipment and supplies used in the simulation included a simulated abdomen (with pelvic structures), vital signs monitor, drapes, laparoscopic tower, and laparoscopic instruments (Box 2). Due to the lack of simulated abdomen or mannequin, the abdomen was constructed from low-cost materials (Box 3) as follows.

A cardboard box of approximate $(50 \mathrm{~cm}$ length $\times 46 \mathrm{~cm}$ width $\times 10 \mathrm{~cm}$ front height $\times 23 \mathrm{~cm}$ back height) was used and double lined with a second layer of cardboard for extra stability and to angle the inner layer in a trapezoid shape in an effort to replicate the shape of a human abdomen and pelvis. If any other low- and high-cost commercially available manikins of abdomen and pelvis are available, they can be substituted for this cardboard box. Large plastic Tupperware or similar storage boxes could also be used for this purpose. Once the abdomen is chosen and constructed, the next step is to assemble internal organs and great vessels connected to a pump. We chose to make our own intestine and to reuse a commercially produced latex uterus model used during another surgical simulation (Applied Medical), but any commercially available or self-made synthetic or meat models would work in this setting. Similarly, greater vessels could be made from Penrose drains and painted over, but we opted to use a commercially available product due to low cost and high fidelity (sold to us at a discount due to minor defects not relevant to the simulation). The latter item, although high fidelity, needs to be covered in moist cloths to maintain proper tissue consistency once opened. This product is made by Lifelike BioTIssue (https://lifelikebiotissue.com/ shop/vascular-surgery/aaa-model). The final component is the pump and tubing used to pump simulated blood during the simulation. Any garden hose pump and extension tubing bought on online marketplaces or hardware stores could be constructed as shown in Supplementary Video 1. Greater vessels are connected to the tubing via a hole in the cephalad end of the box and call components including uterus and bowel and glued and taped to the box (Supplementary Video 1). In this case, we opted not to use a simulated peritoneal layer to make vessels appear retroperitoneal because we found that lack of it did not have an 


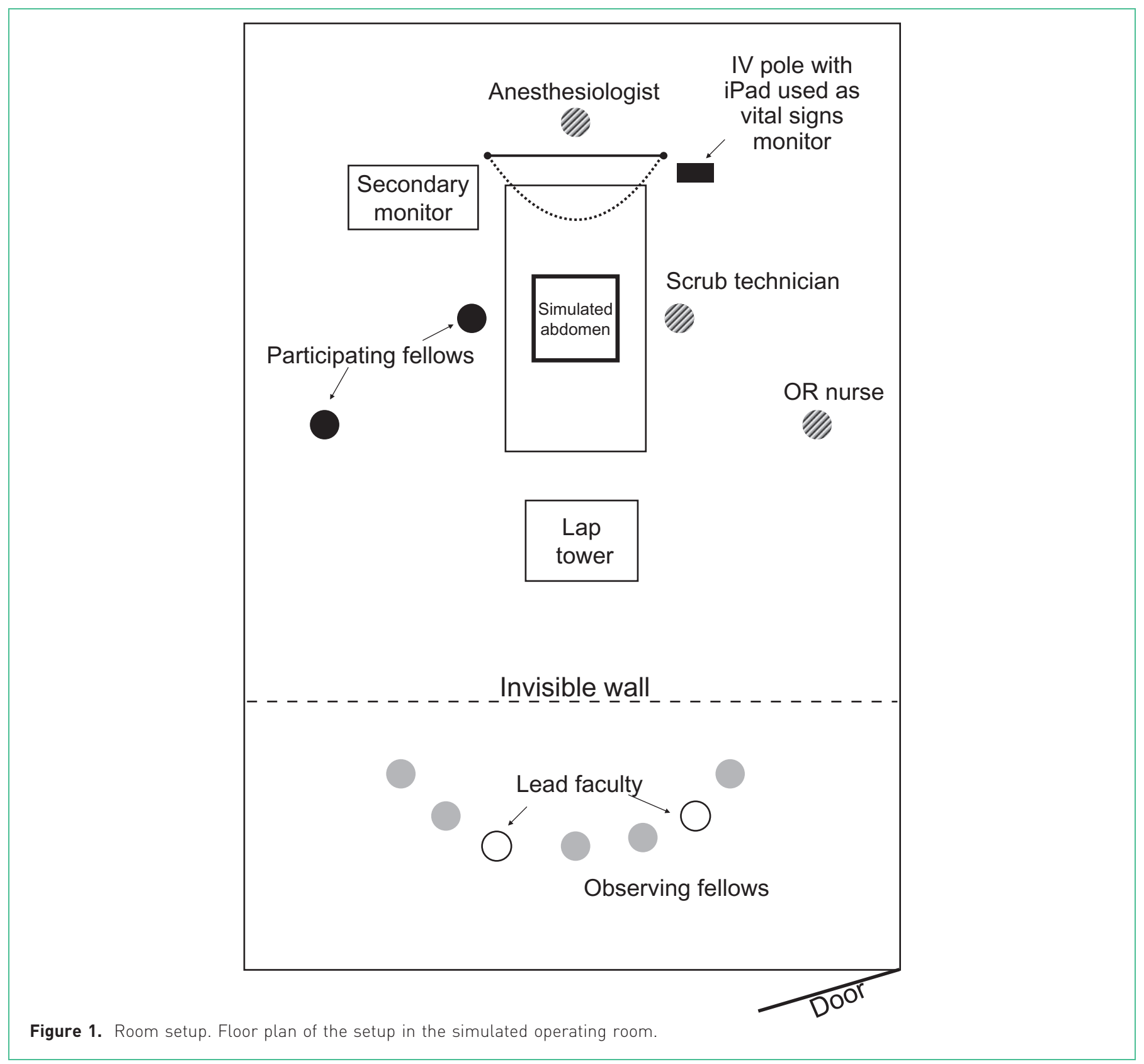

impact on the realism of this technical part, but it can certainly be added if desired. In order to create a receptacle for the pooling simulated blood, we put a funnel in the lower portion of the cephalad part of the box and placed several large trash bags underneath the boxes to catch simulated blood coming out of the funnel or seeping through small defects in the box and lining. This simulated blood can be recycled if the simulation is running multiple times during the day and placed back into the pump. Finally, the top of the box was assembled (Supplementary Video 1). Layers are utilized for stability and realism and consist of large sheets of egg crate foam, a large sheet of felt purchased in a local arts and crafts store and attached to the top of the box with wide tape. We chose to use Applied Kii balloon blunt tip 5- and $10-\mathrm{mm}$ ports because they have fixation points (balloon tips which stabilize the abdominal wall layers during instrument exchange and manipulation). Ports were placed into the abdominal wall before it was attached to the top of the box; after port placement sites were marked based on approximation of the most common placement during the procedure. Before the simulation, the pump was primed and tested to assure proper functionality. Simulated blood was purchased from a commercial vendor (Laerdal) due to the ease of use and transport and high concentration, but any substitute would be acceptable. Supplementary Video 1 demonstrates a laparoscopic view 

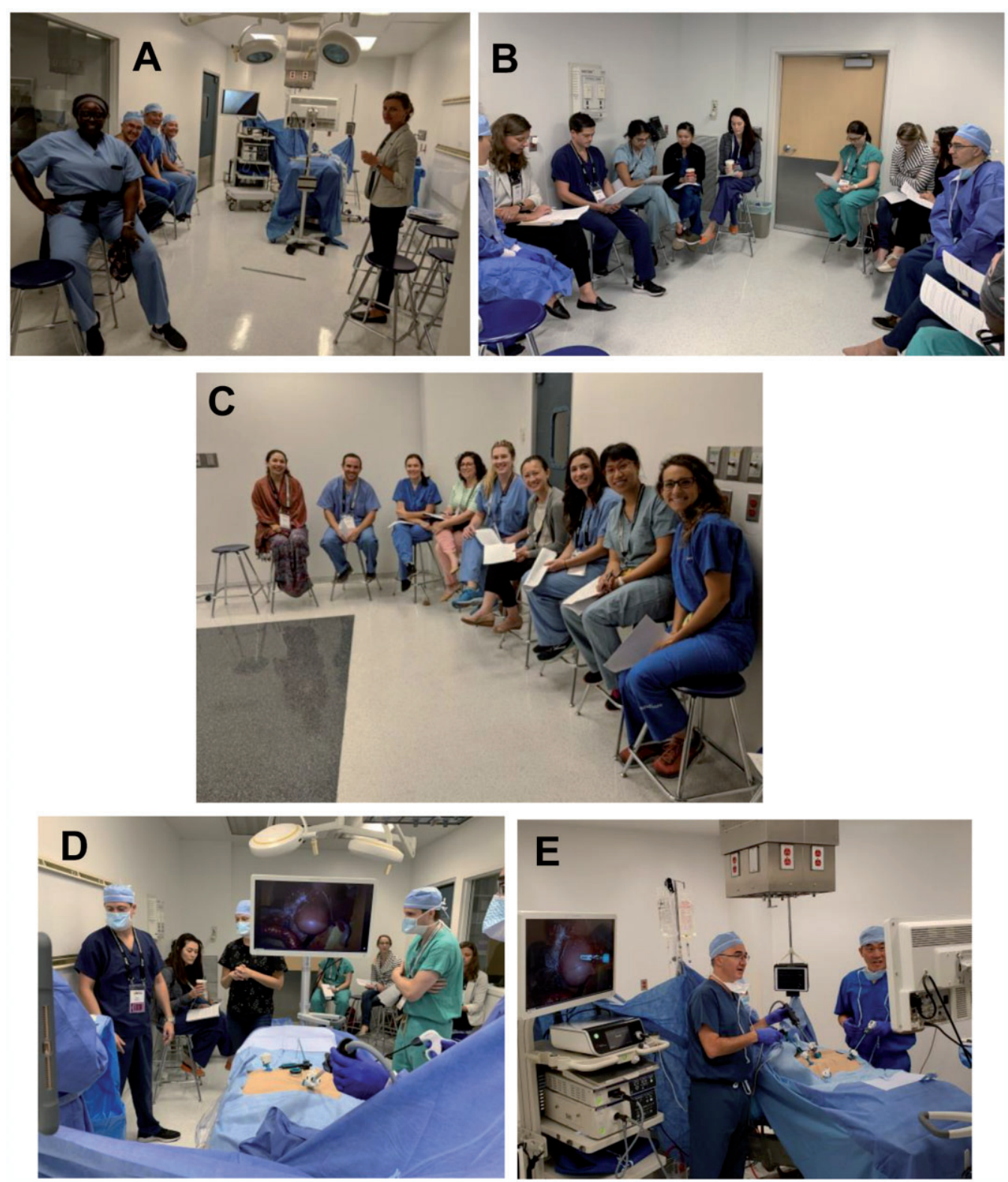

Figure 2. Room setup. (A) The simulation space with setup before participants arrive. (B) The pre-briefing when going over the details of the clinical case before simulation starts. (C) Observers and faculty behind the invisible wall. (D) The simulation in progress as seen by an embedded anesthesiologist from behind the drapes. (E) The simulation in progress as seen by a participant.

of the bleeding vessel with the pump turned on. Due to the need to travel, the entire box including the pump and ports was shipped to the course site from a remote location. Supplementary Video 1, Fig. 3, and Box 3 provide a stepby-step description of the abdomen components and assembly along with the cost. Supplementary Video 1 shows a laparoscopic view of the bleeding vessel as it occurs during the simulation.

\section{The setup}

Fig. 1 shows the layout of the room and Box 2 lists all the items used for the setup. In the encounter space portion of the room, the assembled simulated abdomen was set up on the OR table and covered with drapes sufficiently to represent a human-sized shape underneath the drape. The laparoscopic tower, camera, and instruments were assembled and tested. We opted to use two monitors; one was located near the "head" adjacent to the anesthesia machine and the other was near the foot of the bed. A two-monitor setup was chosen because it improved the laparoscopic view for all participants and faculty in the room. An iPad (or compatible tablet) was used to display vital signs and was taped to an IV pole. The vitals were controlled by the lead faculty from her mobile phone via 
Box 2. Setup checklist

- Laparoscopic tower

- Light cord

- Camera head

- Camera

- Gas tubing (used for props and not for distension, no need for actual gas)

- Laparoscopic instruments

- Suction irrigation ${ }^{\mathrm{a}}$

- Atraumatic graspers (used as a prop)

- Scissors (if available, used as a prop)

- Clip appliers (if available, used as a prop)

- Energy source (e.g. laparoscopic bipolar or ultrasonic device) if available, used as a prop)

- Trocars ${ }^{\mathrm{b}}$

- Instrument table or Mayo stand

- Typical items used as props (lap pads, $4 \times 4$ s, hemostats, scalpels; none of them are actually used during the simulation, only for visual effect)

- OR table (if not available, any table can be used, and simulated abdomen was propped or tilted) with typical items used during surgery

- 2 IV poles to secure (or tape) drapes with IV tubing taped to the trash cans hidden from participant view to simulate administration when asked

- IVF (intravenous fluid) bag æ1 (have 2nd ready to hang if requested by the team)

- Large laparoscopy drape ${ }^{c}$

- Vitals signs monitor

- iPad (or similar tablet) used as vital signs display, controlled by mobile phone via software such as SimMon

- OR attire

- Participants were asked to wear scrubs

- Gowns, gloves, masks for all active participants and embedded persons

${ }^{a}$ If wall suction is not available, portable suction can be utilized; irrigation is not essential. If no suction is available, the simulated abdomen can be tilted and propped up into Trendelenburg position so that simulated blood pulls toward the "head" away from the bleeding site. ${ }^{b}$ See Methods section for details on assembly.

${ }^{c}$ If laparoscopy drape is not available, any typical blue OR drapes can be used and stapled or taped together to make it large enough to cover simulated abdomen with pump, table, and anesthesia area.

an app (SimMon). Efforts were made to optimize the realism of the surgical scenario, including a 1-L bag of crystalloid connected to an IV pole, which drained into a waste receptacle below the bed to simulate IV fluids running into the patient. Additional bags of IV fluids were available if requested by the participants. Four embedded persons (trained faculty) participated in the case: anesthesiologist, consulting gynecologist, scrub technician, and circulating nurse.

\section{The simulation}

A standardized script for orientation of the participants is described in Appendix 2. After orientation, participants were introduced to the simulation space and educated about the functionality of the space (Box 2). Participants were divided into the primary team (two participants), secondary team (up to three participants; used if a primary team calls for help), and observers (remaining trainee team members) who stayed behind the invisible wall.

Next, the participants were presented with the clinical case (Appendix 3). MVI scenario case progression is detailed in Appendix 4 (created for internal use by lead faculty). In brief, participants are expected to recognize an MVI that occurred during laparoscopic entry, resuscitate the patient, perform compression of the bleeding vessel, and call for a vascular surgery consult for vascular repair. A checklist of expected actions was created to serve as a guide for the observers to follow and assist with documenting time, and ultimately aid faculty during the debrief (Boxes 4 and 5). The scenario ended when the expected actions were taken or at a set 15 min cutoff point. 
Box 3. Construction and assembly of simulated abdomen

- Materials needed for construction of the abdomen

- Cardboard box (plastic storage bin or similar container can be used instead)

- Box cutter

- Paper liner

- Paints for the inside of the cardboard box to make it look like flesh

- Press and seal (or any other similar plastic lining)

- Eggcrate

$\circ$ Large piece of felt

- Duct tape and masking tape

- Scissors

- Funnel

- Trocars

- Greater vessels

- Simulated bowel

- Simulated uterus

- Pump

- Simulated blood
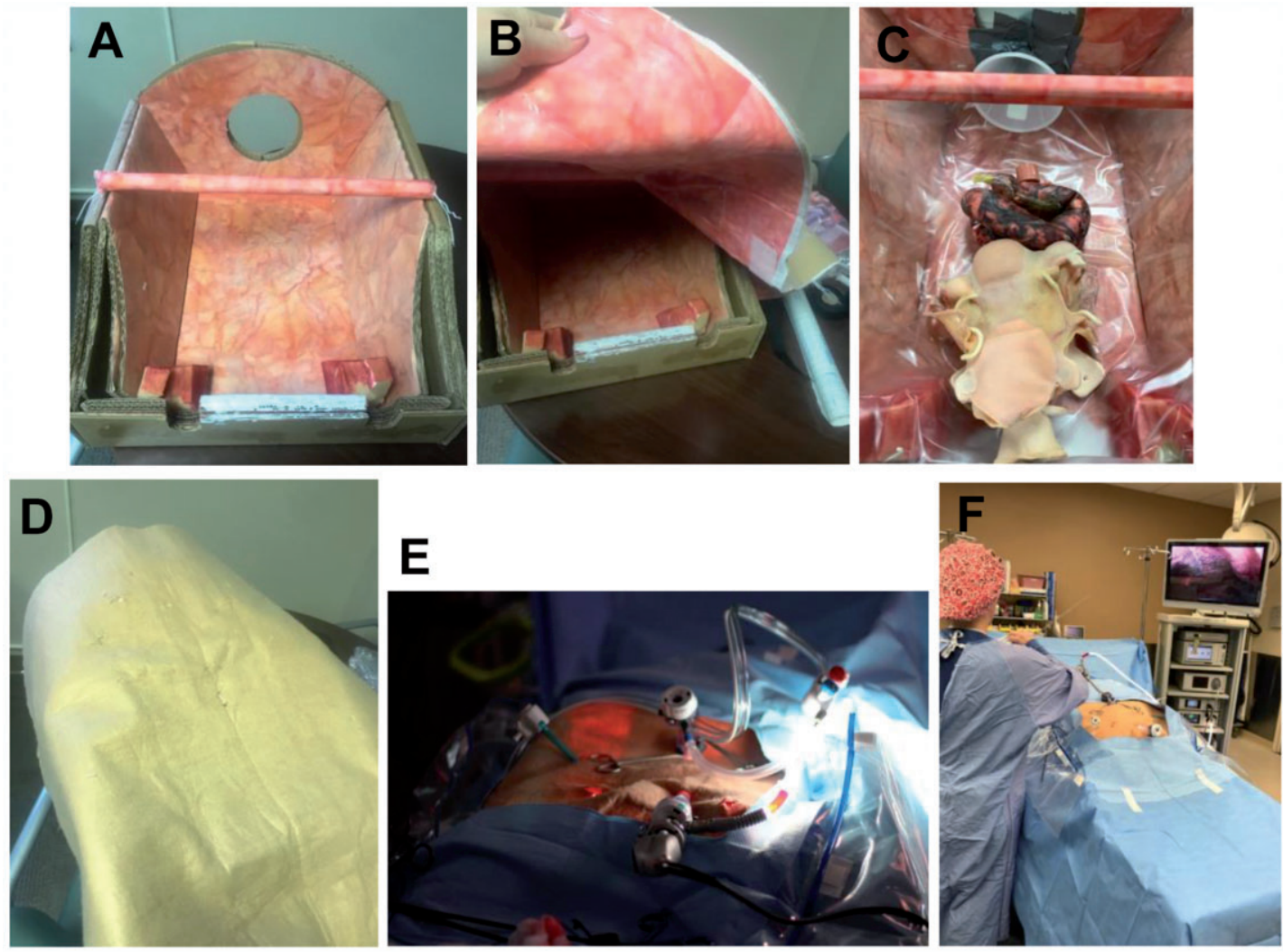

Figure 3. Construction and setup of the simulated abdomen. (A) Carboard box with liner; (B) cover for the box; (C) box with internal organ inserts; (D) fully assembled abdomen; (E) abdomen with ports during simulated surgery; (F) view of the abdomen, tower, and surgeon. 
Box 4. Action checklist

- Focused assessment once entering the OR

- Identify the patient as an individual with life-threatening intraoperative bleeding secondary to operative complication

- Communicate effectively with the primary surgeon and with team

- Consult appropriately with vascular surgery

- Activate massive transfusion protocol

- Obtain additional IV access

- Fluid resuscitation and volume expanders

- Make conservative efforts to stop the bleeding

- Recognize need to convert to midline vertical laparotomy

- Send stat labs when staffing allows

Box 5. Teaching aid for debrief

- Technical aspects:

- How did injury occur?

- During retroperitoneal dissection, significant retroperitoneal fibrosis from deeply infiltrating endometriosis obscured normal anatomy and the external iliac artery was inadvertently injured

- Basic tenets of vascular injury during surgery

- Compression will control almost any vascular injury without causing further complication

- Compression-patience-resuscitation (vascular CPR) approach:

- Mistake is to rush to fix the problem; blind clamping, blind sutures lead to worse problems

- Blood loss is to be anticipated so resuscitation is crucial before attempts to isolate and repair the injury

- Need proximal and distal control once patient is stable so the injury can be repaired

- For venous injury, direct compression above and below is best option

- Clamping will tear the vein and make it worse

- Once converted to laparotomy

- Immediate manual compression of the area

- DO NOT attempt any type of clamping, energy, blind stitches, staples, etc. (ureteral injury, worsening hemorrhage occurs if either attempted)

- Compression controls the bleeding

- Communicate with anesthesia regarding a large arterial injury and will need blood products

- Initiate massive transfusion protocol

- Maintain compression on the area until the patient is adequately resuscitated before beginning exploration

- Non-technical aspects

$\circ$ Identify that life-threatening hemorrhage is occurring

- Laparotomy

- Must communicate with anesthesia their concern and plan to convert

- Request that anesthesia begin resuscitation

- Call for assistance

- Another surgeon

- Additional anesthesia and nursing personnel

- Additional OR staff

- Vascular surgery/trauma team 
Lead faculty made an announcement when the scenario was over, and the entire group returned to the debriefing side of the room. Lead faculty facilitated the debrief via PEARLS and Advocacy/Inquiry methods.

\section{Assessment of participant perception}

A survey was designed to ask faculty and participants about their simulation experience. Faculty and participants were asked to fill out this survey anonymously before and after the simulation (Supplementary Material) with the goal of evaluating their previous experience of MVI simulation and perceptions of the value of the simulation as a training exercise. Pre- and post-simulation survey responses were compared using the McNemar test. Median and interquartile ranges were calculated on responses. All fellows were eligible to participate in the simulation.

Institutional Review Board approval was obtained to analyze participant surveys (IRB\#2019-10888; approval 31 December 2019).

\section{Results}

Simulation took place on 11 August 2019. Results of the faculty and fellow surveys are presented in Tables 2-5. A total of 34 fellows (median age, 31.5 years) and 8 faculty (median age, 37.5 years) responded. Fellows reported a median of $8 \mathrm{~h}$ per year spent participating in simulation team training and a median of $5 \mathrm{~h}$ per year spent teaching in this context. All fellows believed that simulation training is both an essential part of clinical practice and patient safety. Thirty-two (94.1\%) thought that simulation team training should be a part of the annual MIGS fellow boot camp. Thirty-two (94.1\%) also thought that this training should be part of the fellowship curriculum in their respective institutions. Before the simulation, 33 (97.1\%) of the fellows desired to learn more about simulation training and curriculum development. After the simulation, all fellows felt that knowledge gained could be transferred to their clinical setting, felt more confident in responding to a critical intraoperative event, learned techniques to communicate more effectively during an emergency, and felt that the simulation content was relevant to their training and clinical practice. Most of the fellows felt more confident in managing intraoperative hemorrhage (median score, 5; interquartile range, 4-5) after the simulation.

Faculty reported a median of 4 years in practice and a median of $10 \mathrm{~h}$ per year spent teaching via simulation that involved team training. They reported $0 \mathrm{~h}$ per year spent as participants in simulation team training. Most of the faculty $(85.7 \%-100 \%)$ believed that simulation training was an essential part of clinical practice, an important part
Table 2. Faculty survey results, faculty demographics ( $n=8)$

\begin{tabular}{ll}
\hline Age (years), median (IQR) & $37.5(34.5,40.5)$ \\
Years in practice, median (IQR) & $4.0(3.5,8.0)$ \\
$\begin{array}{c}\text { Hours per year spent participating in simulation } \\
\text { team training, median (IQR) }\end{array}$ & $0.0(0.0,0.0)$ \\
$\begin{array}{c}\text { Hours per year spent teaching via simulation team } \\
\text { training, median (IQR) }\end{array}$ & $10.0(9.0,17.5)$ \\
$\begin{array}{c}\text { Hours per year spent participating in standardized } \\
\text { patient-based simulation training, median (IQR) }\end{array}$ & $0.0(0.0,0.0)$ \\
$\begin{array}{c}\text { Hours per year spent teaching in standardized } \\
\text { patient-based simulation training, median (IQR) }\end{array}$ & $2.0(0.0,12.5)$ \\
\hline
\end{tabular}

IQR, interquartile range.

of patient safety, and an activity that should be part of the annual MIGS fellow boot camp. Most (85.7\%) also expressed a desire to learn more about simulation training and simulation-based curriculum development and thought that it should be part of fellowship curricula in their respective institutions. Half of the faculty members expressed an interest in the development of a simulation scenario/case, debriefing techniques, evaluation of trainees/students.

\section{Discussion}

This pilot study has demonstrated the feasibility of surgical crisis simulation training for MIGS fellows, critical for management of MVI during laparoscopy. The participants responded favorably, indicating that this simulation met learning objectives (CRM techniques and medical and technical knowledge), and felt more confident in managing intraoperative hemorrhage. We also noted that participants believed that simulation was important to clinical practice and wanted to learn more about simulation training.

In terms of feasibility and favorable response by participants, the findings of this study are similar to earlier work by Moorthy et al. ${ }^{2}$ Moorthy and colleagues conducted a study on a simulation-based approach to the management of femoral vein injury in which they described their simulation and model and used rating scales to assess variation in videotaped technical and non-technical skills. Simulation is increasingly important in rare surgical scenarios to encourage mental modeling and improve efficiency when time is of the essence.

Strengths of this study include the construction of a novel, low-cost, self-made, reusable, multi-purpose abdominal simulator and the ability to set up and conduct a simulation in space with no simulation equipment. Use of embedded persons assisted with buy-in of the participants and realism of the scenario. Fellows attending this surgical course 
Table 3. Faculty pre- and post-simulation survey results $(n=7)$

\begin{tabular}{|c|c|c|c|}
\hline & Pre, $n(\%)$ & Post, $n(\%)$ & $P$ value \\
\hline Believe that simulation training is an essential part of clinical practice & $7(100.0)$ & $6(85.7)$ & Non-est. \\
\hline Believe that simulation training is an important part of patient safety & $6(85.7)$ & $7(100)$ & Non-est. \\
\hline Think simulation team training should be part of AAGL MIGS fellow bootcamp & $7(100)$ & $7(100)$ & Non-est. \\
\hline Think simulation team training should be part of fellowship curriculum in your institution & $6(85.7)$ & $5(71.4)$ & 0.3173 \\
\hline Desire to learn more about simulation training and simulation-based curriculum development & $6(85.7)$ & $6(85.7)$ & Non-est. \\
\hline $\begin{array}{l}\text { Would attend a postgraduate course or round table discussion at the AAGL annual meeting regarding } \\
\text { simulation training and simulation-based curriculum development }\end{array}$ & $6(85.7)$ & $6(85.7)$ & Non-est. \\
\hline Interest in developing a simulation scenario/case & $5(71.4)$ & $6(85.7)$ & 0.3173 \\
\hline Interest in understanding available technology for simulation & $5(71.4)$ & $5(71.4)$ & Non-est. \\
\hline Interest in debriefing techniques & $5(71.4)$ & $6(85.7)$ & 0.3173 \\
\hline Interest in evaluation of trainees/students & $4(57.1)$ & $4(57.1)$ & Non-est. \\
\hline
\end{tabular}

Most of the $P$ values are non-estimable either because every participant endorsed the item at pre or at post, or because all of the participants answered the same way at both pre and post. The three non-significant $P$ values indicate that the pre-post change of 5 to 6 or 6 to 5 participants endorsing the item is not a statistically significant change.

* McNemar's test.

Table 4. Fellow survey results ( $N=34)$, fellow demographics

$\begin{array}{ll}\text { Age (years), median (IQR) } & 31.5(31.0,33.0) \\ \text { Years in fellowship, median (IQR) } & 2.0(2.0,2.0) \\ \text { Hours per year spent participating in simulation team training, median (IQR) } & 8.0(1.0,20.0) \\ \text { Hours per year spent teaching via simulation team training, median (IQR) } & 5.0(0.0,10.0) \\ \text { Hours per year spent participating in standardized patient-based simulation training, median (IQR) } & 0.0(0.0,0.0) \\ \text { Hours per year spent teaching in standardized patient-based simulation training, median (IQR) } & 0.0(0.0,0.0) \\ \text { Believe that simulation training is an essential part of clinical practice, } n(\%) & 34(100.0) \\ \text { Believe that simulation training is an important part of patient safety, } n \text { (\%) } & 34(100.0) \\ \text { Think simulation team training should be part of AAGL MIGS fellow bootcamp, } n \text { (\%) } & 32(94.1) \\ \text { Think simulation team training should be part of the fellowship curriculum in your institution, } n(\%) & 32(94.1) \\ \text { Desire to learn more about simulation training and simulation-based curriculum development, } n(\%) & 33 \text { (97.1) } \\ \text { Would attend a postgraduate course or round table discussion at the AAGL annual meeting } & 29 \text { (85.3) } \\ \text { regarding simulation training and simulation-based curriculum development, } n(\%) & 21(61.8) \\ \text { Interest in developing a simulation scenario/case, } n \text { (\%) } & 21(61.8) \\ \text { Interest in understanding available technology for simulation, } n \text { (\%) } & 16(47.1) \\ \text { Interest in debriefing techniques, } n \text { (\%) } & 20 \text { (58.8) } \\ \text { Interest in evaluation of trainees/students, } n \text { (\%) } & \end{array}$

IQR, interquartile range.

practiced in many geographic areas and different practice settings, making our findings more generalizable. Limitations of our study include the inability to study the effect of this simulation on performance and clinical outcomes. The former would require work to develop assessment tools, which was beyond the scope of this project. The latter is not likely to be feasible due to the low incidence of MVI in clinical practice. In addition, the survey used in this study was created by the authors because it had not been studied previously. With regard to the simulated abdomen, our focus was not to create a high-fidelity task trainer to teach technical skills for vascular repair, but we do support this level of training because the surgical approach to MVI differs based on abdominal location. Although our pre-reading materials did contain detailed descriptions of vascular repair, we did not include this skill as an objective in our simulation due to time constraints. Integrating this model into future work would be useful because it would allow 
Table 5. Post-simulation fellow survey $(N=34)$

\begin{tabular}{|c|c|}
\hline Question & $\begin{array}{l}\text { Median } \\
\text { (IQR) }\end{array}$ \\
\hline 1. The faculty clearly communicated the objectives of the simulation scenarios & $5.0(5.0,5.0)$ \\
\hline 2. The faculty supported a safe learning environment that encouraged active learning & $5.0(5.0,5.0)$ \\
\hline 3. The knowledge gained through the simulation experience can be transferred to the clinical setting & $5.0(5.0,5.0)$ \\
\hline 4. The simulation scenarios allowed me to use critical-thinking skills & $5.0(5.0,5.0)$ \\
\hline 5. The faculty provided constructive feedback and encouraged discussion after the simulation scenarios & $5.0(5.0,5.0)$ \\
\hline 6. I have more confidence in responding to a critical event in the future & $5.0(5.0,5.0)$ \\
\hline 7. I have learned techniques to communicate more effectively during a critical event & $5.0(5.0,5.0)$ \\
\hline 8. The major vascular injury session has given me confidence in managing intraoperative hemorrhage & $5.0(4.0,5.0)$ \\
\hline 9. I recommend that the AAGL boot camp include such simulation sessions in future programs & $5.0(5.0,5.0)$ \\
\hline 10. Topic and content covered in this session were relevant to my training and clinical practice & $5.0(5.0,5.0)$ \\
\hline 11. Format (simulation, hands-on skills) was appropriate for the content & $5.0(5.0,5.0)$ \\
\hline 12. How prepared did you feel for managing an intraoperative hemorrhage before this session? & $3.0(2.0,3.0)$ \\
\hline 13. How prepared do you feel for managing an intraoperative hemorrhage after this session? & $4.0(4.0,4.0)$ \\
\hline
\end{tabular}

$\mathrm{IQR}$, interquartile range.

gynecologic surgeons to practice this skill in a hands-on and individual manner. Finally, while working on this project, we faced challenges similar to the current educational system with regard to flaws, limitations, and lack of critical resident and fellow education in OBGYN. We hope that our community will be able to start working on those challenges to improve education for our trainees and care for our patients.

Future research could explore the impact of this simulation on performance in a simulated and clinical setting and the impact on clinical outcomes. The latter, however, would be difficult to measure given the rarity of the event and might require proxy metrics. Skill transferability to other clinical scenarios and skill retention could also be explored. Another aspect that requires further study is the barriers to wider use and implementation of team-based simulation in gynecologic surgery. Although a culture of simulation has permeated obstetrics, partially motivated by the financial burden of medicolegal costs related to complications, this has not yet occurred in gynecology, where lawsuit payouts are much lower. Aside from financial incentives, barriers to the widespread use of simulation are multifactorial and are seen across disciplines and specialties and could be further explored in this setting of gynecologic surgery more specifically. ${ }^{11,12}$

In conclusion, we developed an MVI simulation scenario in MIGS that was inexpensive and easy to conduct. We hope this work will aid clinicians and educators in the implementation of simulation in their institutions and lead to further research in this area and contribute to culture change.

\section{Conflict of interest}

The authors report no conflict of interest and nothing to disclose.

\section{Funding}

No funding sources to disclose.

\section{Acknowledgement}

We thank Caitlin Martins, MD, for assistance with model construction.

\section{Supplementary material}

Supplementary Video 1. Pump assembly, abdomen construction, operating room set up and simulation of bleeding from a major vessel. Available from https://youtu.be/_DnNJSzFf4c

Supplementary File. Surveys. Available online at https://doi. org/10.5281/zenodo.5827671.

\section{References}

1. Yu SP, Cohen JG, Parker WH. Management of hemorrhage during gynecologic surgery. Clin Obstet Gynecol 2015; 58(4): 718-731. https://doi.org/10.1097/GRF.0000000000000147.

2. Moorthy K, Munz Y, Forrest D, Pandey V, Undre S, Vincent $\mathrm{C}$, et al. Surgical crisis management skills training and assessment: a simulation[corrected]-based approach to enhancing 
operating room performance. Ann Surg 2006; 244(1): 139147. https://doi.org/10.1097/01.sla.0000217618.30744.61.

3. AHRQ Quality Indicators - Guide to Patient Safety Indicators. Rockville, MD: Agency for Healthcare Research and Quality, 2003. Version 2.1, Revision 2 (October 22, 2004). AHRQ Pub. 03-R203. Available from: https://www.qualityindicators.ahrq. gov/Downloads/Modules/PSI/V21/psi_guide_rev2.pdf. Accessed 6 January 2020.

4. Smith KK, Gilcreast D, Pierce K. Evaluation of staff's retention of ACLS and BLS skills. Resuscitation 2008; 78(1): 59-65. https://doi.org/10.1016/j.resuscitation.2008.02.007.

5. Helmreich RL. On error management: lessons from aviation. BMJ 2000; 320(7237): 781-785. https://doi.org/10.1136/bmj. 320.7237 .781 .

6. TeamSTEPPS. Available from: https://www.ahrq.gov/team stepps/index.html. Accessed 10 May 2020.

7. ACOG Committee Opinion No. 487: Preparing for clinical emergencies in obstetrics and gynecology. Obstet Gynecol 2011; 117(4): 1032-1034. https://doi.org/10.1097/AOG.0b013e31821922eb.

8. Arriaga AF, Bader AM, Wong JM, Lipsitz SR, Berry WR, Ziewacz JE, et al. Simulation-based trial of surgical-crisis checklists. N Engl J Med 2013; 368(3): 246-253. https://doi. org/10.1056/NEJMsa1204720.

9. Eppich W, Cheng A. Promoting Excellence and Reflective Learning in Simulation (PEARLS): development and rationale for a blended approach to health care simulation debriefing. Simul Healthc 2015; 10(2): 106-115. https://doi.org/10. 1097/SIH.0000000000000072.

10. Rudolph JW, Simon R, Dufresne RL, Raemer DB. There's no such thing as "nonjudgmental" debriefing: a theory and method for debriefing with good judgment. Simul Healthc 2006; 1(1): 49-55. https://doi.org/10.1097/01266021-200600110-00006.

11. Hosny SG, Johnston MJ, Pucher PH, Erridge S, Darzi A. Barriers to the implementation and uptake of simulationbased training programs in general surgery: a multinational qualitative study. J Surg Res 2017; 220: 419-426.e412. https://doi.org/10.1016/j.jss.2017.07.020.

12. De Tina A, Chau A, Carusi DA, Robinson JN, Tsen LC, Farber MK. Identifying barriers to implementation of the national partnership for maternal safety obstetric hemorrhage bundle at a tertiary center: utilization of the Delphi method. Anesth Analg 2019; 124(9): 1045-1050. https://doi.org/10. 1213/ANE.0000000000003451.

\section{Appendix 1. Resources for participants and faculty}

\section{Faculty}

Brett-Fleegler M. Debriefing assessment for simulation in healthcare. Simul Healthc 2012; 7(5): 288-294. https://doi. org/10.1097/SIH.0b013e3182620228
Eppich W, Cheng A. Promoting Excellence and Reflective Learning in Simulation (PEARLS): development and rationale for a blended approach to health care simulation debriefing. Simul Healthc 2015; 10(2): 106-115. https://doi. org/10.1097/SIH.0000000000000072

Parker W, Wagner W. Gynecologic surgery and the management of hemorrhage. Obstet Gynecol Clin N Am 2010; 37: 427-436. https://doi.org/10.1016/j.ogc.2010.05.003

Kuroki L, Mutch D. Control of pelvic hemorrhage (chapter 19). In: Jones HW, Rock JA (Eds). Te Linde's Operative Gynecology, 11th edn. Wolters Kluwer; 2015.

Rudolph JW, Simon R, Dufresne RL, Raemer DB, Rudolph JW. There is no such thing as 'non-judgmental' debriefing; a theory and method of debriefing with good judgment. Simul Healthc 2006; 1(1): 49-55. https://doi.org/10. 1097/01266021-200600110-00006

\section{Participants}

Parker W, Wagner W. Gynecologic surgery and the management of hemorrhage. Obstet Gynecol Clin N Am 2010; 37: 427-436. https://doi.org/10.1016/j.ogc.2010.05.003

Kuroki L, Mutch D. Control of Pelvic Hemorrhage (chapter 19). Jones HW, Rock JA (Eds). Te Linde's Operative Gynecology, 11th edn. Wolters Kluwer; 2015.

\section{Appendix 2. Standardized script for partici- pant orientation to the simulation during introduction}

\section{Confidentiality agreement}

- Program directors (residency or fellowship) will not be given a formal report about individual performance here. Instead, the simulation today will be used to provide participants with formative feedback on developing intraoperative crisis management skills. You might see us taking notes during the session, but that is only so that we could use that information for discussion during the debrief. We ask that faculty and learner participants do not discuss each other's individual performance outside of this group. Please nod if you agree.

- We worked hard preparing today's case in hopes of making this a valuable educational experience for you, and we ask you today to keep the case content of these cases confidential because your colleagues may be taking this course in the future, and knowing the scenario ahead of time would take away from their experience. 
- We realize that participants can view simulation as another "test", a stressful situation even if not graded. Moreover, we believe in the basic assumption that everyone here is intelligent, doing their best, and wants to improve. We understand that people may not behave the same way in a simulation as they would in real life, and we acknowledge that limitation. However, we believe it is still valuable, because simulation is a very powerful way of teaching complex medical concepts effectively and is an opportunity to practice your communication, evaluation and management skills in a safe environment.

- We try our best to make the simulation a safe learning environment, so we ask you in advance to keep comments that other participants make and their actions confidential, and to refrain from discussing the case and details of the case with anyone outside this room. Please nod if you agree.

\section{Fiction contract}

- We do our best to make the scenarios as engaging as possible, but we know that simulation is not exactly the same as a real patient encounter. Because simulation is not real, yet valuable, we ask that you do your best to act as if it is real because this will enhance everyone's learning.

\section{Orientation to simulation}

- Today, you (two fellows as a primary team) will participate in an OR simulation in which you are called into the OR for an intraoperative consult; the primary surgeon will be requesting assistance with a laparoscopic left salpingo-oophorectomy for unanticipated stage IV endometriosis.

- Additional members of your OR team will include scrub and circulating nurses and anesthesiologist. You may request three additional learners (fellows and/or residents) to assist the OR team if needed by the primary OR team and consultant surgical team.

- The three additional learners will be available to the two consultant surgeons as support. The group of learners will be given a few minutes before the start of the simulation to "pre-brief" as a team to establish roles. Learners will be given the opportunity to volunteer for lead and support roles. If there are no volunteers, learners' roles will be assigned randomly.

\section{Physical and functional orientation to space}

- Participants are shown OR, vital signs monitor, surgical field, towers and instruments.

- Functional details:

- Please do not leave the encounter space. If you need to leave based on what is happening in the encounter (such as to call for help), please notify the simulation facilitator to assist you with this task;

- Please do not cut or manipulate the abdomen and pelvis. If you would like to perform a laparotomy for example, please verbalize you are doing so;

- If you would like to call the operator to speak with the blood bank, radiology, or to call another provider, please use the imaginary phone (participants were shown how to do that: face facilitator and speak to them as if they were speaking on a phone);

- Stay in your role (i.e. if you are PGY-5 in your home institution, you are the same during this encounter);

- The simulation will last approximately $15 \mathrm{~min}$.

\section{Appendix 3. Clinical case description pre- sented to participants before the start of the clinical encounter}

You (MIGS fellow team) are paged into the operating room by your obstetrics and gynecology colleague. MIGS attending is tied up in clinic off-site $20 \mathrm{~min}$ away and is not immediately available; she asked you to go to the OR assess the situation for her.

The primary surgeon (OBGYN generalist) is asking for laparoscopic assistance during surgery she is performing on her patient. She encountered extensive pelvic adhesions during a case.

While responding to your page for assistance, you quickly review the patient's preoperative history and physical, which describes a healthy 39-year-old G1P1 female who presented for pelvic pain and recurrent left adnexal mass. Imaging was consistent with recurrent endometrioma. Below is the summary of her clinical course.

- Past medical history: significant for dysmenorrhea, dyschezia, and dyspareunia

- Past surgical history: significant for laparoscopic appendectomy and laparoscopic ovarian cystectomy for previous left endometrioma

- Medications: none 
- Allergy to penicillin (severe hives)

- Preoperative physical exam: pulse, 67; blood pressure, 110/36; respiratory rate, 12; height, $1.63 \mathrm{~m}$; weight, $50 \mathrm{~kg}$

- Abdominal exam: tender to palpation in left lower quadrant, no rebound or guarding

- Bimanual exam: retroverted uterus, immobile left adnexal mass, and left uterosacral tethering

- Labs: white blood cell count, 9000; hemoglobin, 11.2; hematocrit, 35; platelets, 160,000

- Transvaginal ultrasonography reveals $12 \mathrm{~cm}$ left ovarian cyst, suspicious for endometrioma.

After appropriate discussion, she consented to an outpatient laparoscopic left salpingo-oophorectomy, excision of endometriosis.

\section{Appendix 4. Scenario and case progression}

The simulation starts with learners walking into the OR after being called in for an urgent consult. Vitals: heart rate, 91; blood pressure, 127/89.

Part 1: Decision to convert to laparotomy, control of hemorrhage, and recognition of vascular injury

- Upon opening the abdomen, a large amount of arterial bleeding is encountered from the left retroperitoneal area. Patient is becoming increasingly hypotensive despite crystalloid resuscitation.

- If an attempt is made at blind clamping, the use of energy, blind stitches, staples, etc. then simulation faculty continues to simulate further hemorrhage and ureteral injury.

- Vitals: heart rate,119; blood pressure, 101/78; then pulse, 129, blood pressure 91/62.

\section{Part 2: Resuscitation}

- At the start of the case the patient only has one IV; will have minor blood antibody issues.

- Patient received two units of packed red blood cells through a rapid infuser (if requested) and after $10 \mathrm{~min}$ of compression has stabilized with a systolic blood pressure of $100 \mathrm{mmHg}$ and pulse of 70 beats/min. With removal of compression, recurrent arterial hemorrhage occurs from a hole in the external iliac artery.

\section{Part 3: Consult vascular surgery}

- A laceration can now be seen well, and no other site of bleeding is identified. With compression, the patient is stable. 